\title{
Análisis de las sentencias judiciales en la práctica de la cardiología resueltas en segunda instancia en España entre 1992 y 2007.
}

Santiago Sáez, Andrés, Perea Pérez, Bernardo, Albarrán Juan, María Elena, Labajo González, Elena, Anadón Baselga, María José y Almendral Garrote, Jesús.

Cita:

Santiago Sáez, Andrés, Perea Pérez, Bernardo, Albarrán Juan, María Elena, Labajo González, Elena, Anadón Baselga, María José y Almendral Garrote, Jesús (2012). Análisis de las sentencias judiciales en la práctica de la cardiología resueltas en segunda instancia en España entre 1992 y 2007. Revista española de Cardiología, 65 (9), 801-806.

Dirección estable: https://www.aacademica.org/elenalabajogonzalez/9 ARK: https://n2t.net/ark:/13683/pcQr/Daa

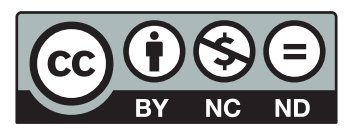


Artículo original

\title{
Análisis de las sentencias judiciales en la práctica de la cardiología resueltas en segunda instancia en España entre 1992 y 2007
}

\author{
Andrés Santiago-Sáez ${ }^{\mathrm{a}, \mathrm{b}, *}$, Bernardo Perea-Pérez ${ }^{\mathrm{b}}$, Maria Elena Albarrán-Juan ${ }^{\mathrm{b}}$, Elena Labajo-González ${ }^{\mathrm{b}}$, \\ María José Anadón-Baselga ${ }^{\mathrm{b}}$ y Jesus Almendral-Garrote ${ }^{\mathrm{c}}$ \\ a Servicio de Medicina Legal, Hospital Clínico San Carlos, Madrid, España \\ ${ }^{\mathrm{b}}$ Escuela de Medicina Legal, Facultad de Medicina, Universidad Complutense, Madrid, España \\ ${ }^{c}$ Unidad de Electrofisiología Cardiaca y Arritmología Clínica, Grupo Hospital de Madrid, Universidad CEU San Pablo, Madrid, España
}

Historia del artículo:

Recibido el 27 de septiembre de 2011

Aceptado el 2 de febrero de 2012

On-line el 7 de junio de 2012

\section{Palabras clave:}

Sentencias judiciales

Reclamaciones

Condenas

Indemnizaciones

Síndrome coronario

\section{RES U M E N}

Introducción y objetivos: El incremento de la judicialización de los asuntos sanitarios ha aumentado el interés hacia las demandas contra médicos. El objetivo es analizar las sentencias relacionadas con la práctica de la cardiología en España en época reciente.

Métodos: Se han analizado las 1.899 sentencias judiciales sanitarias emitidas en España en segunda instancia o posteriores durante el periodo de 1992 a 2007. La ficha de datos consta de 25 variables administrativas, clínicas y judiciales. Al estudio descriptivo, se añaden comparaciones estadísticas entre variables cuya posible relación se juzga de interés.

Resultados: Existen 32 sentencias en ámbito cardiológico, y son condenatorias el 31\%, con cuantías nunca superiores a los 365.000 euros. Se ha cuadruplicado el número de denuncias en 2000-2007 comparado con 1992-1999. La patología más frecuente es el síndrome coronario (50\%). Hay relación estadística $(p=0,004)$ entre tipo de profesional implicado y causa de la demanda: las causas más frecuentes (error diagnóstico y/o mala praxis) afectaron a los no cardiólogos, mientras que las demandas por defecto de información (que alcanzan el 9,5\%) recaen mayoritariamente en cardiólogos. El fallecimiento del paciente se produjo en el $75 \%$ de los casos.

Conclusiones: Aunque la cardiología tiene "bajo riesgo" de demanda judicial comparada con otras especialidades, la actividad litigante aumentó notablemente en años recientes. Las causas de demanda se relacionaron con el tipo de profesional implicado, y destacan el error diagnóstico y la mala praxis por su frecuencia y el defecto de información por su novedad.

(c) 2012 Sociedad Española de Cardiología. Publicado por Elsevier España, S.L. Todos los derechos reservados.

Analysis of Judgments in the Practice of Cardiology Resolved on Appeal in Spain Between 1992 and 2007

\footnotetext{
A B S T R A C T
}

Introduction and objectives: The increase in the prosecution of health issues in Spain has increased the interest in legal claims against physicians. Our objective is to analyze the judgments issued in relation to cardiology practice in Spain in recent years.

Methods: We analyzed the 1899 sanitary judicial sentences issued in Spain in the second instance or later during the period 1992 to 2007 . The data sheet includes 25 administrative, clinical, and judicial variables. In addition to a descriptive study, comparative analysis was performed on selected variables. Results: There were 32 sentences in the field of cardiology, 31\% decided for the plaintiff and with an award amount never exceeding 365000 euros. The most frequent clinical diagnosis is coronary syndrome (50\%). There is a significant statistical relationship $(P<.004)$ between physician specialty and reason for the claim: the most frequent causes (misdiagnosis and malpractice) affected predominantly noncardiologists, while defects in information or informed consent ( $9.5 \%$ of cases) affected cardiologists exclusively. Patient death occurred in $75 \%$ of cases.

Conclusions: Despite cardiology being a "low-risk discipline" compared to others, the litigant activity has increased dramatically in recent years. Causes for the claims are related to the specialty of the involved physician; misdiagnosis and/or malpractice and defects in information should be emphasized, the former for its frequency and the latter for its novelty.

Full English text available from: www.revespcardiol.org

(c) 2012 Sociedad Española de Cardiología. Published by Elsevier España, S.L. All rights reserved. 


\section{INTRODUCCIÓN}

El incremento de la judicialización de los asuntos sanitarios en España afecta a todas las especialidades, sean quirúrgicas o médicas, hospitalarias o extrahospitalarias. Durante el periodo de 1995 a 1998, se presentaron contra el Sistema Nacional de Salud 932 reclamaciones. Los errores médicos costaron al extinto INSALUD (Instituto Nacional de la Salud) 4,3 millones de euros por indemnizaciones a 95 pacientes (una media de 47.000 euros por paciente). Por especialidades, medicina de urgencia supuso el $23,8 \%$ de las reclamaciones; ginecología y obstetricia, el $18,2 \%$ y traumatología, el 11,2\% ${ }^{1}$. Estos datos concuerdan con el estudio realizado por Torre $^{2}$, de la compañía de seguros Willis, con datos del reaseguro de los servicios públicos de salud en España durante el periodo 2005-2007. Un estudio del National Health Service británico informó de que durante el periodo 1999-2000 se cerraron 3.254 reclamaciones, con un coste de 462 millones de euros ${ }^{3}$.

Una consecuencia inmediata de esta situación puede ser la práctica sistemática de la medicina defensiva, que se aleja de la relación fluida entre médico y paciente, basada en la confianza mutua y con una orientación bioética que potencia la propia autonomía del paciente. Sin embargo, un análisis de los factores que motivan una demanda y de los condicionamientos que influyen en las decisiones judiciales puede ayudar a los especialistas en cardiología a valorar determinadas prácticas clínicas de forma especial, extremando las precauciones ante los signos que puedan dar lugar a una demanda judicial.

En este artículo pretendemos determinar las características de las sentencias emitidas contra médicos que han realizado una práctica cardiológica, considerando todas las variables que pueden tener alguna influencia tanto en el planteamiento de la reclamación como en la resolución del proceso judicial. Por lo tanto, el objetivo es establecer las principales características desde el punto de vista médico-legal, sobre todo en cuanto a si hay algún tipo de condena y su cuantía. De este modo, los resultados podrán facilitar una práctica más segura de la especialidad, tanto para el paciente como para el médico, ya que existen algunas particularidades propias de dicha especialidad que conviene señalar, y dado que de su práctica puede derivarse una responsabilidad profesional.

Por último queremos resaltar que no existe en la literatura médica de los últimos años ningún estudio similar, que haga referencia específica a la especialidad de cardiología en España.

\section{MÉTODOS}

\section{Origen del material y criterios de inclusión}

El presente estudio se ha realizado sobre el "Archivo de sentencias judiciales sanitarias de la Escuela de Medicina Legal», de la Universidad Complutense de Madrid. Este archivo contiene 1.899 sentencias judiciales de segunda o última instancia (depuradas de un total de 6.300), referidas a actuaciones sanitarias emitidas por las jurisdicciones civil, penal y contencioso-administrativa durante el periodo comprendido entre enero de 1992 y junio de 2007. El citado archivo se nutre de las sentencias publicadas por la base de datos de Westlaw Aranzadi, que durante el periodo de estudio recogía la totalidad de sentencias judiciales de segunda instancia o posterior publicadas durante el periodo de estudio, utilizando para ello las siguientes voces: responsabilidad profesional, mala praxis, negligencia, sanitario, reclamación, daño corporal, denuncia, demanda, error médico e imprudencia. Tenemos que aclarar que la jerarquía jurisdiccional española se establece por su función procesal; podemos distinguir, por un lado, los órganos de instancia - primera o única- que son los que resuelven los asuntos por primera vez; pero en determinados casos cabe una segunda instancia, es decir, un nuevo enjuiciamiento pleno, ante un órgano superior estratificado de la siguiente forma: Audiencia Provincial (máximo órgano en la provincia), Tribunal Superior de Justicia (máximo órgano en la comunidad autónoma), Tribunal Supremo (máximo órgano nacional), Audiencia Nacional (entiende de asuntos nacionales de terrorismo, crimen organizado, tráfico de armas o de drogas, etc.). Las sentencias de primera instancia no se publican uniformemente en ninguna base de datos, el desacuerdo en la sentencia es lo que motiva acudir a una segunda instancia. Existe una excepción para la vía de reclamación contencioso-administrativa, ya que se acude directamente al Tribunal Superior de Justicia de la comunidad autónoma sin pasar por la primera instancia.

El archivo contiene 32 sentencias que se ha considerado para este estudio con base en los siguientes criterios de inclusión: a) sentencia referida a un médico especialista en cardiología, debido al ejercicio de su especialidad, o sentencia referida a un médico en la que no consta su especialidad, pero en la que la maniobra reclamada judicialmente corresponde a la especialidad de cardiología, según definición y maniobras incluidas en el anexo a la orden SCO/1259/2007, de 13 de abril, por la que se aprueba y publica el programa formativo de la especialidad de cardiología, y b) texto de la sentencia en el que figure el tratamiento reclamado, las circunstancias y el lugar en que se produjo, sus consecuencias y la resolución judicial.

\section{Variables recogidas}

En la ficha de recogida de datos se han implementado las siguientes variables, clasificadas según el ámbito a que corresponden:

- Variables administrativas: clave de identificación en la base de Aranzadi (referencia); jurisdicción en la que se dirime la demanda civil, penal o contencioso-administrativa; fecha de la sentencia; comunidad autónoma donde se produce la demanda; tribunal en el que se resuelve la demanda, y ámbito de actividad del profesional demandado.

- Variables clínicas: edad del paciente, sexo del paciente, síntoma dominante y enfermedad diagnosticada.

- Variables judiciales: número de imputados; si hay un cardiólogo implicado o no; tipo de profesional: cardiólogo u otro tipo de especialista o estamento (no cardiólogo); motivo de la demanda; resultado del daño demandado; cuantía económica de la demanda; cuantía de la indemnización en caso de condena, y si hubo condena o no.

\section{Análisis estadístico}

En primer lugar, se ha realizado un estudio descriptivo simple de las variables recogidas, mediante porcentajes. En lo referente a las comunidades autónomas y a los ámbitos de actividad del profesional demandado, con objeto de "corregir» de algún modo el número de sentencias respecto a la actividad médica realizada, se presenta el número de camas hospitalarias existentes según datos del Ministerio de Sanidad, Política Social e Igualdad actualizado a enero de 2011. De esta manera obtenemos el índice de sentencias por cada 100.000 camas hospitalarias (tabla 1 ). En los conjuntos de dos variables cuya distribución pudiera estar relacionada y esa relación pudiera ser de interés, se ha realizado un contraste de variables mediante tablas de contingencia usando la prueba de la $\chi^{2}$ utilizando el programa estadístico SPSS 19. 
Tabla 1

Variables administrativas

\begin{tabular}{|c|c|c|}
\hline Variable & Categorías & \\
\hline \multirow[t]{3}{*}{ Jurisdicción $(n=32)$} & Civil & $24(75)$ \\
\hline & Penal & $4(12,5)$ \\
\hline & Contencioso-administrativa & $4(12,5)$ \\
\hline \multirow{2}{*}{$\begin{array}{l}\text { Fecha de la sentencia } \\
\quad(n=32)\end{array}$} & 1992-1999 & $6(18,8)$ \\
\hline & $2000-2007$ & $26(81,2)$ \\
\hline \multirow{9}{*}{$\begin{array}{l}\text { Comunidad autónoma } \\
\text { (número de camas } \\
\text { hospitalarias) } \\
(n=27)^{\mathrm{a}}\end{array}$} & Andalucía (22.319) & $1(3,1)[4,5]$ \\
\hline & Principado de Asturias (4.182) & $1(3,1)[23,9]$ \\
\hline & Castilla y León (9.579) & $3(9,4)[31,3]$ \\
\hline & Cataluña (33.641) & $10(31,3)[29,7]$ \\
\hline & Galicia (10.443) & $1(3,1)[9,6]$ \\
\hline & Comunidad de Madrid (21.236) & $5(15,6)[23,5]$ \\
\hline & Región de Murcia (4.534) & $2(6,3)[44,1]$ \\
\hline & País Vasco (8.330) & $2(6,3)[24]$ \\
\hline & Comunidad Valenciana (13.724) & $2(6,3)[14,6]$ \\
\hline \multirow[t]{3}{*}{ Tribunal $(n=32)$} & Audiencia Provincial & $23(71,9)$ \\
\hline & Tribunal Superior de Justicia & $4(12,5)$ \\
\hline & Tribunal Supremo & $5(15,6)$ \\
\hline \multirow{3}{*}{$\begin{array}{l}\text { Ámbito asistencial } \\
\quad \text { (número de camas) } \\
(n=29)^{\mathrm{b}}\end{array}$} & Público (106.658) & $19(65,5)[17,8]$ \\
\hline & Privado (52.164) & $8(27,6)[15,3]$ \\
\hline & Mutua (1462) & $2(6,9)[136,7]$ \\
\hline
\end{tabular}

Datos presentados como n (\%) [índice de sentencias/1.000 camas hospitalarias].

a Cinco casos se resolvieron en el Tribunal Supremo.

b Número de camas en España: 160.284.

\section{RESULTADOS}

\section{Estudio descriptivo}

La tabla 1 recoge las variables administrativas. En la jurisdicción civil, se ha resuelto la mayoría (75\%) de las sentencias. Se observa un incremento en el número de sentencias a lo largo de los años de estudio. En los últimos 8 años (2000 a 2007) se han producido 26 sentencias, lo que supone un $430 \%$ de aumento con respecto a las 6 sentencias ocurridas en los primeros 8 años del estudio (1992 a 1999). De las 17 comunidades autónomas, se han producido sentencias sólo en 9. Aunque en valor absoluto en Cataluña y la Comunidad de Madrid se han producido más demandas, el índice de sentencias por cada 100.000 camas hospitalarias es máximo en la Región de Murcia, Castilla y León y Cataluña. Aunque la mayoría (65\%) de los casos han ocurrido en el ámbito público, el índice de sentencias por cada 100.000 camas hospitalarias es similar en los ámbitos público y privado.

La tabla 2 refleja las variables clínicas. La mayoría son varones, y la patología que más frecuentemente origina la demanda es el síndrome coronario, que supone al menos el 50\% de todas las demás patologías agrupadas. En cuanto al síntoma principal, los datos son más dispersos.

En la tabla 3 se presentan las variables judiciales. Aunque predomina la implicación de un único profesional (casi 60\%), es más frecuente que este no sea cardiólogo. Está implicado un cardiólogo sólo en un tercio de los casos. El hecho más frecuentemente imputado como motivo de la denuncia es la mala praxis, junto al error diagnóstico; la falta de medios, algo no imputable al médico, es la segunda causa más frecuente. Llama la atención la inexistencia de demandas por error terapéutico. En la mayoría de las ocasiones, el resultado que motiva la demanda es la muerte. La distribución de la cuantía inicialmente demandada resulta similar a la de la indemnización finalmente sentenciada, y
Tabla 2

Variables clínicas

\begin{tabular}{llc} 
Variable & Categorías & $\mathrm{n}(\%)$ \\
\hline Edad del paciente $(n=8)^{*}$ & $<18$ años & 0 \\
\hline & 18 a 40 años & $4(50)$ \\
\hline & 41 a 65 años & $2(25)$ \\
\hline & $>65$ años & $2(25)$ \\
\hline Sexo $(n=31)$ & Varón & $21(67,7)$ \\
\hline Síntoma $(n=30)$ & Mujer & $10(32,3)$ \\
\hline & Dolor torácico típico & $4(13,3)$ \\
\hline & Dolor torácico atípico & $3(10)$ \\
\hline Patología del paciente $(n=32)$ & Síntomas no cardiacos & $10(33,3)$ \\
\hline & Otros síntomas cardiovasculares & $13(43,3)$ \\
\hline & Síndrome coronario & $1(3,1)$ \\
\hline & Malformación & $16(50)$ \\
\hline & Insuficiencia cardiaca & $1(3,1)$ \\
\hline & Vascular & $2(6,3)$ \\
\hline & Cardiopatía (sin especificar $)$ & $8(25)$ \\
\hline & & $4(12,5)$ \\
\hline
\end{tabular}

* En 24 casos no consta.

Tabla 3

Variables judiciales

\begin{tabular}{|c|c|c|}
\hline Variable & Categorías & $\mathrm{n}(\%)$ \\
\hline \multirow{2}{*}{$\begin{array}{l}\text { Número de profesionales } \\
\text { implicados }(n=31)\end{array}$} & Uno & $19(61)$ \\
\hline & Más de uno & $12(39)$ \\
\hline \multirow{2}{*}{$\begin{array}{l}\text { Estaba implicado un } \\
\text { cardiólogo }(n=28)\end{array}$} & Sí & $10(35,7)$ \\
\hline & No & $18(64,3)$ \\
\hline \multirow{5}{*}{$\begin{array}{l}\text { Tipo de profesional } \\
\text { demandado }(n=32)\end{array}$} & Cardiólogo & $6(19)$ \\
\hline & Cardiólogo + otro facultativo & $3(9)$ \\
\hline & Cardiólogo + otro estamento & $1(3)$ \\
\hline & Otro profesional & $18(56)$ \\
\hline & No consta & $4(12)$ \\
\hline \multirow[t]{4}{*}{$\begin{array}{l}\text { Hecho imputado motivo } \\
\quad \text { de la demanda }(n=32)\end{array}$} & $\begin{array}{l}\text { Defecto de información, } \\
\text { comunicación } \\
\text { o consentimiento }\end{array}$ & $3(9,3)$ \\
\hline & $\begin{array}{l}\text { Error diagnóstico } \\
\text { y mala praxis }\end{array}$ & $18(56,25)$ \\
\hline & Error seguimiento & $3(9,3)$ \\
\hline & Falta de medios & $8(25)$ \\
\hline \multirow{2}{*}{$\begin{array}{l}\text { Resultado del daño } \\
\quad \text { demandado }(n=32)\end{array}$} & Muerte & $24(75)$ \\
\hline & Lesiones & $8(25)$ \\
\hline \multirow{2}{*}{$\begin{array}{l}\text { Cuantía económica } \\
\text { de la demanda }(n=18)\end{array}$} & $\geq 60.000$ euros & $15(83)$ \\
\hline & $<60.000$ euros & $3(17)$ \\
\hline \multirow{2}{*}{$\begin{array}{l}\text { Cuantía de la indemnización } \\
(n=10)\end{array}$} & $\geq 60.000$ euros & $7(70)$ \\
\hline & $<60.000$ euros & $3(30)$ \\
\hline \multirow[t]{2}{*}{ Condena $(n=32)$} & Sí & $10(31)$ \\
\hline & No & $22(69)$ \\
\hline
\end{tabular}

son mayoritarias las indemnizaciones superiores a 60.000 euros. La cuantía media de indemnización por caso fue de 113.506,31 euros, sin tener en cuenta las costas judiciales. La sentencia fue condenatoria en la tercera parte y exculpatoria en el resto (fig. 1).

\section{Relaciones estadísticas entre las variables analizadas}

Hay asociación estadísticamente significativa entre imputación por síndrome coronario y no ser especialista en cardiología 


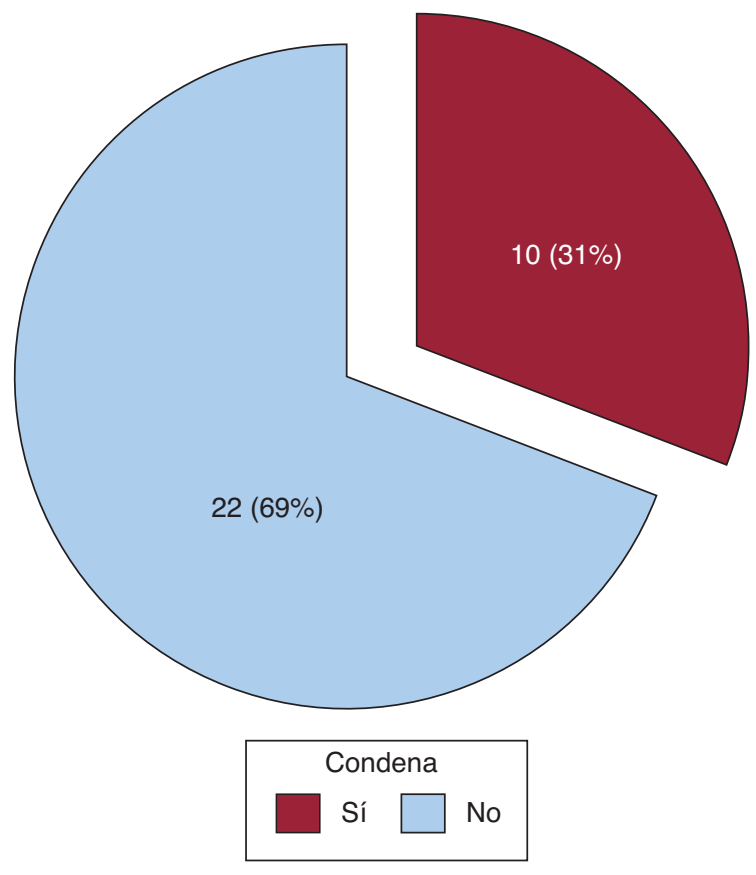

Figura 1. Sentencias condenatorias.

$(\mathrm{p}=0,044)$. Al agrupar la patología y dicotomizarla en «síndrome coronario» frente a "otras», esta asociación se hace más fuerte ( $p=0,002$ ) (fig. 2): 13 de las 14 demandas por síndrome coronario afectaron a profesionales no cardiólogos. El contraste de la variable patología dicotomizada frente a la variable demanda económica dicotomizada (mayor o menor de 60.000 euros) presenta una tendencia $(p=0,058)$ a que las patologías que no son síndrome coronario sean reclamadas con más frecuencia por un importe mayor de 60.000 euros.

Hay relación estadísticamente significativa entre que el implicado sea cardiólogo o no y el tipo de acto imputado $(p=0,004)$ (tabla 4): en 14 de las 15 imputaciones por mal praxis o error diagnóstico, el profesional implicado no era cardiólogo, mientras que las 3 demandas por defecto de información se produjeron sobre cardiólogos. Además la misma variable «cardiólogo implicado" tiende a asociarse con la presencia de síntomas cardiovasculares, lo que tiene mucha significación clínica, pero no llega a alcanzar significación estadística.

En el cruce de las variables tipo de patología y existencia de condena, destaca la ausencia de condenas en dos tercios de los casos de síndrome coronario. Sin embargo, al cruzar la primera variable con la cuantía de la condena, los casos de este grupo (síndrome coronario) que son condenados lo hacen mayoritariamente $(67 \%)$ en el grupo de $>60.000$ euros, con una cuantía media por caso de 67.250 euros.

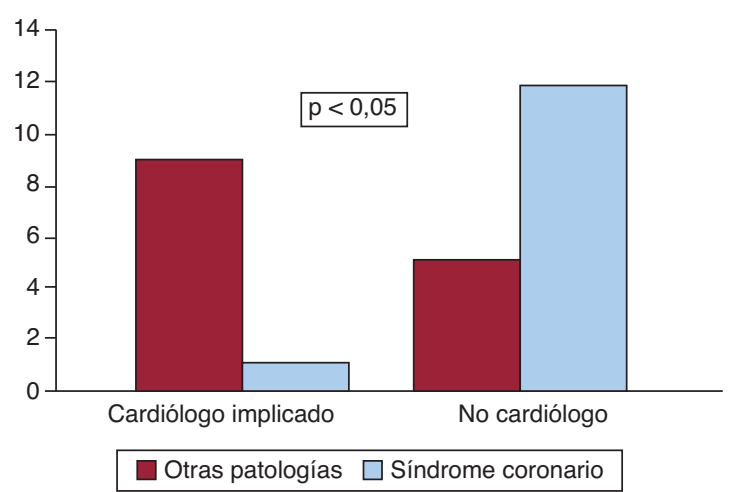

Figura 2. Contraste de la variable "cardiólogo implicado" con el tipo de patología.

\section{DISCUSIÓN}

La descripción de las sentencias realizadas en relación con procesos cardiológicos a lo largo de 16 años nos muestra principalmente que estos procesos tienen "bajo riesgo» judicial; que este riesgo, sin embargo, se ha cuadruplicado en años recientes; que hay marcadas diferencias entre comunidades autónomas; que la probabilidad de una resolución condenatoria es baja pero su cuantía económica es alta (aunque nunca rebasa los 365.000 euros), y que la mala praxis y el error diagnóstico son las causas más frecuentes de condena, pero afectan más a profesionales no cardiólogos, mientras que aparece una proporción no despreciable de condenas por déficit de información, lo que, curiosamente, afecta más a los cardiólogos.

\section{Comparación con otros países y otras especialidades, ámbito de aplicación}

La cardiología no es una especialidad de alto riesgo judicial, y se sitúa en puestos muy alejados de la cabeza de las diferentes series nacionales e internacionales, aunque los estudios son escasos y normalmente proceden de fuentes interesadas (compañías de seguros, asociaciones de pacientes, etc.). El estudio del periodo 1995-2000 del INSALUD no recoge esta especialidad entre las 12 más reclamadas ${ }^{1}$. De hecho, las 32 sentencias encontradas en relación con la práctica cardiológica representan tan sólo el $1,63 \%$ de todas las sentencias. Aunque el número de sentencias pueda ser mayor porque aquí recogemos sólo aquellas en segunda o posterior instancia, estas representan las de mayor envergadura y condenatorias por la costumbre habitual de recurrir por alguna de las partes a instancias superiores si la resolución no es satisfactoria. En el estudio realizado sobre las quejas presentadas ante la Comisión Nacional de Arbitraje Médico ${ }^{4}$ procedentes de México, Distrito Federal, de un total de 1.925 quejas, se asignaron a la especialidad de cardiología 49 , lo que supone un $2,45 \%$, porcentaje similar al de nuestra serie.

Tabla 4

Relación entre tipo de profesional implicado y motivo de la demanda*

\begin{tabular}{|c|c|c|c|c|}
\hline & $\begin{array}{l}\text { Mala praxis } \\
\text { o error diagnóstico }\end{array}$ & $\begin{array}{l}\text { Defecto de información } \\
\text { o comunicación }\end{array}$ & $\begin{array}{l}\text { Falta de } \\
\text { medios }\end{array}$ & $\begin{array}{l}\text { Error de } \\
\text { seguimiento }\end{array}$ \\
\hline Cardiólogo & 1 & 3 & 4 & 2 \\
\hline Otros profesionales & 14 & 0 & 3 & 1 \\
\hline Total & 15 & 3 & 7 & 3 \\
\hline
\end{tabular}

En 4 casos no consta el tipo de profesional implicado. La distribución del motivo de la demanda muestra diferencias estadísticamente significativas ( $\mathrm{p}=0,004$ ) según el profesional implicado sea cardiólogo o no. 
Las tendencias detectadas en cuanto a la reclamación cardiológica en nuestro país muestran un perfil que se caracteriza por: a) ser de orden civil (frente a penal); b) dirimirse en la Audiencia Provincial (frente al Tribunal Supremo); c) ser más frecuente en los últimos 8 años (frente a los 8 anteriores), y d) variar considerablemente en su incidencia de una comunidad autónoma a otra.

Las cantidades económicas demandadas superan casi siempre los 60.000 euros, pero no existen condenas superiores a 365.000 euros, lo que supone situarse dentro del límite de los 600.000 euros que habitualmente se suscribe en las pólizas de seguro por responsabilidad profesional como máximo de indemnización por siniestro en la mayoría de las compañías. Durante el decenio 19811991, un estudio realizado por la Universidad de Texas reveló indemnizaciones, en casos denunciados sobre todo por suicidios y abusos sexuales a pacientes, en la franja entre 200.000 y 375.000 euros $^{5}$, cifras que, actualizadas a fecha de hoy, serían muy superiores a lo aquí observado.

El número de sentencias condenatorias de un tercio resulta inferior a la de otras especialidades médicas ${ }^{6}$.

\section{Aspectos clínicos y motivos de la demanda}

En cuanto a los motivos de la denuncia destaca que no exista ningún caso por error terapéutico, a pesar de la trascendencia que supone el uso de la muy variada farmacopea cardiológica y del uso vital que esta adquiere en determinadas ocasiones. Que las primeras causas sean errores diagnósticos y mala praxis y la frecuencia de fallecimiento en los casos demandados coinciden con un estudio realizado en Francia en 2007, donde se hace una valoración de las especiales medidas que se debe tomar con los pacientes, ya desde el servicio de urgencias de los centros sanitarios, para prevenir la muerte ${ }^{7}$.

Los síndromes coronarios identifican el diagnóstico del 50\% de los casos, lo que evidencia la potencial conflictividad de este tipo de cuadros y lo importante que resulta extremar su manejo tanto médico como institucional.

En una minoría importante de las sentencias se apreciaba un déficit en la información facilitada al paciente o sus familiares o en el documento de consentimiento informado. En este sentido, hay que recordar que la documentación del consentimiento informado no exime de las demandas, pero su ausencia las agrava. Y debe existir un adecuado formato, claro y conciso del consentimiento ${ }^{8-10}$. El proceso de una buena información y el otorgamiento del consentimiento se considera un elemento relevante en la práctica asistencial, es una obligación exigible al profesional de la medicina y forma parte integrante de la Lex Artis, cuyo incumplimiento determina por sí mismo la obligación reparadora (sentencias del Tribunal Supremo de 22 de octubre de 1993, 17 de enero de 1994 y 24 de abril de 1994). Por ello, sus deficiencias incurren generalmente en responsabilidad, lo que viene a afianzar la corriente de la relación entre una práctica segura y la buena comunicación el paciente. En este sentido, el simple documento legal que hace que el paciente refleje por escrito que ha sido informado y que consiente el procedimiento no evita las demandas. De hecho, no es la firma del documento lo que constituye el consentimiento informado, sino el asentimiento del paciente a una información detallada. La firma del documento es sólo la constancia de esta. El consentimiento informado no tiene validez, o al menos eficacia, si no existe una relación fluida entre el paciente y el médico. A este respecto, Scott y Kim, del Departamento de Psiquiatría de la Universidad de Michigan, insiste en los aspectos no sólo legales sino también éticos del consentimiento ${ }^{11}$.

\section{Tipo de profesional y tipo de sentencia}

En dos terceras partes se imputa el daño a un solo profesional, lo que significa que el denunciante suele identificar a un profesional como responsable de los resultados. En cuanto al tipo de profesional denunciado, los cardiólogos como único profesional representan una minoría (20\%).

En cuanto a la cualificación profesional del médico reclamado, hay clara asociación entre el tipo de patología reclamada (síndrome coronario, que supone el 50\% de las reclamaciones) y el motivo del acto imputado (error diagnóstico y mala praxis) con el hecho de no ser especialista en cardiología. Aunque esto pueda deberse a la frecuente implicación de profesionales no cardiólogos en el tratamiento de los síndromes coronarios, también puede reflejar una percepción de mayor nivel de pericia en los cardiólogos, a quienes, por tanto, no se atribuye mala praxis o errores diagnósticos. Por el contrario, debe resultar preocupante para la comunidad cardiológica que las demandas por defecto de información se concentren en los cardiólogos.

El riesgo judicial de la cardiología, aunque pequeño, podría ocasionar una reacción del especialista, que se plantearía una medicina defensiva, dilatando en el tiempo la puesta en marcha de medidas diagnósticas o terapéuticas, enfatizando el riesgo de algunos procedimientos $\mathrm{y}$, posiblemente, distanciándose del paciente. En Inglaterra un cuestionario enviado a médicos psiquiatras identificó que un $75 \%$ de los profesionales había realizado medicina defensiva en el mes anterior ${ }^{12}$. Sin embargo, la actitud defensiva no parece ser la solución a este problema. Una mala comunicación con el paciente incrementa enormemente el riesgo de litigio ${ }^{13}$. Un análisis de las demandas hechas en Estados Unidos ${ }^{14}$ demostró que un factor independiente que distinguía entre que se demandara o no era el número de minutos que el médico dedica a la entrevista, una media de 15,0 entre los demandados y 18.3 entre los no demandados. Factores involucrados en la forma de la relación entre el médico y el enfermo, como el tono de voz, también parecen ser determinantes.

\section{CONCLUSIONES}

El número de sentencias contra la práctica cardiológica en segunda o posterior instancia en España es escasa hasta la fecha, aunque se ha cuadruplicado en los últimos años respecto al primer periodo del estudio. Tan sólo un tercio de las resoluciones son condenatorias. Las indemnizaciones económicas son altas, aunque nunca superan los 365.000 euros, cuantía que se encuentra dentro de los límites marcados habitualmente por las pólizas de seguros por cada siniestro.

Los motivos fundamentales de las reclamaciones son el error diagnóstico y la posible mala praxis, y hay asociación estadística entre este hecho y no ser especialista en cardiología. En un tercio de las sentencias interviene un especialista en cardiología. No existe ningún caso sentenciado por error terapéutico.

La demanda se produce típicamente a raíz de un fallecimiento, y principalmente por patología relacionada con un síndrome coronario.

Existe un $10 \%$ de casos en los que se aprecia déficit en el proceso de información o en el otorgamiento del consentimiento.

\section{AGRADECIMIENTOS}

El presente trabajo ha sido posible gracias a la aportación del análisis estadístico que ha realizado D. Pedro Cuesta, del Departamento de Apoyo a la Investigación de la Universidad Complutense de Madrid. 


\section{CONFLICTO DE INTERESES}

\section{Ninguno.}

\section{BIBLIOGRAFÍA}

1. INSALUD. Memoria Estadística INSALUD. Madrid: Ministerio de Sanidad y Consumo; 1999.

2. Torre D. El seguro de responsabilidad civil. En: Actas del XII Encuentro de Responsabilidad Sanitaria. Madrid: Unidad Editorial; 2008.

3. Fenn P. Counting the cost of medical negligence. BMJ. 2002;325:233-4.

4. Valdés-Salgado R, Molina-Leza J, Solís-Torres C. Aprender de lo sucedido. Análisis de las quejas presentadas ante la Comisión Nacional de Arbitraje Médico. Salud Pública de México. 2001;43:444-54.

5. Wagner KD, Pollard R, Wagner Jr RF. Malpractice litigation against child and adolescent psychiatry residency programs 1981-1991. J Am Acad Child Adolesc Psychiatry. 1993;32:462-5.

6. Perea-Pérez B, Santiago-Sáez A, Labajo-González ME, Albarrán-Juan ME. Análisis de las consecuencias médico-legales de las reclamaciones judiciales en pacientes. Estudio comparativo de los casos de traumatología, ginecología y obstetricia, anestesia y reanimación y odontoestomatología. Trauma. 2009; 20:264-8.

7. Franchitto N, Mathur A, Franchitto L, Gavarri L, Telmon N, Rougé D. Emergency suicide care in France: the psychiatrist's liability as seen in case law. Int J Law Psychiatry. 2007;30:472-9.

8. De los Reyes López M, Iñíguez Romo A, Goicolea de Oro A, Funes López B, Castro Beiras A. El consentimiento informado en cardiología. Rev Esp Cardiol 1998;51:782-96.

9. De Pablo Zarzosa C, Maroto Montero JM. El consentimiento informado en la rehabilitación cardíaca. Rev Esp Cardiol. 1999;52:362.

10. De los Reyes López M, Iñíguez Romo A. El consentimiento informado en la rehabilitación cardíaca. Réplica. Rev Esp Cardiol. 1999;52:363.

11. Scott Y, Kim H. Evidence-based ethics for neurology and psychiatry research. NeuroRx. 2004;1:372-7.

12. Passmore K, Leung WC. Defensive practice among psychiatrists: a questionnaire. Survey. Postgrad Med J. 2002;78:671-3.

13. Lester G, Smith S. Listening and talking to patients. A remedy for malpractice suits? West J Med. 1993;158:268-72.

14. Levinson W, Roter DL, Mullooly JP, Dull VT, Frankel RM. Physician-patient communication. The relationship with malpractice claims among primary care physicians and surgeons. JAMA. 1997;277:553-9. 